\title{
Analytical and numerical studies of asteroid perturbations on solar system planet dynamics
}

\author{
A. Fienga and J.-L. Simon \\ Institut de mécanique céleste et de calcul des éphémérides (IMCCE) - Observatoire de Paris, UMR 8028 du CNRS, \\ 77 avenue Denfert-Rochereau, 75014 Paris, France \\ e-mail: fienga@imcce.fr \\ Received 26 April 2004 / Accepted 5 August 2004

\begin{abstract}
We present the first results based on the new analytical solution of planetary motion, VSOP2002b (Variations Seculaires des Orbites Planetaires) and on our new planetary numerical integration. After the presentation of this new solution, we discuss the impact of the perturbations induced by the main belt asteroids on short term dynamics of inner planets. We used our two planetary solutions, one analytical (VSOP2002b) and one numerical, to estimate such impacts. Comparisons are made with JPL DE405 and new contributions to Mercury and Venus orbits arise. Same results are found with the two methods and the accuracy limit of the new analytical solution is extended a few tens of meters over three decades for the Mars and the Earth-Moon Barycenter (EMB) orbits.
\end{abstract}

Key words. ephemerides - planets and satellites: general - minor planets, asteroids - celestial mechanics

\section{Introduction}

Accuracy limits in the ephemerides of Mars and the EarthMoon barycenter (EMB) imposed by uncertainties in asteroid modeling were described and analysed previously (Williams 1984; Standish \& Fienga 2002). Different alternative models were also investigated (i.e. Krasinsky et al. 2002). In this work, the perturbations from 300 main belt asteroids onto all planets were taken into account by simultaneous numerical integration of the equations of motion of all planets and 300 asteroids. However, no specific study was done concerning the impact of the gravitational perturbations of the main belt asteroids upon the other inner planets, Mercury and Venus. In the JPL DE ephemerides, asteroid perturbations were taken into account for Mars and the EMB. In this paper, we will show that the perturbations of the main belt asteroids are non negligible upon the Mercury and Venus orbits. Two approaches have been used for this study. The first approach is analytical and based on the VSOP2002 solution of Bretagnon (2002).

The second approach is a direct numerical integration of the asteroid perturbations over all the nine planets and the solar system barycenter. In the second section, we will introduce the two methods and show that the two approaches give very comparable results. In the third section, we will discuss the consequences of new results.

\section{Two approaches}

\subsection{Analytical model}

\subsubsection{The VSOP2002 solution}

Our analytical solution comes from the lastest works of Bretagnon (2002). Starting from the VSOP2000 solution of Moisson \& Bretagnon (2001), Bretagnon performed 15 additional iterations with a numerical precision 10 times better than in VSOP2000. The main characteristics of that solution are the following:

- The eight major planets and the five asteroids Ceres, Pallas, Vesta, Iris and Bamberga (hereafter referred to as the 5bigs) have their orbits analytically computed during the same process.

- Perturbations of the Moon on the eight major planets are introduced during the iteration process.

- Relativistic corrections have been included (Moisson \& Bretagnon 2001).

- The solution is fitted to the JPL numerical integration DE403 (Standish et al. 1995), over the epoch [1890, 2000].

- The used variables are the elliptic variables $a, \lambda, k=$ $e \cos \varpi, h=e \sin \varpi, q=\sin (i / 2) \cos \Omega, p=\sin (i / 2) \sin \Omega$ where $a$ is the semi-major axis, $\lambda$ the mean longitude, $e$ the eccentricity of the orbit, $\varpi$ the longitude of the perihelion, $i$ the inclination about the ecliptic J2000 and $\Omega$ the longitude of a node. 
Table 1. Maximum differences between VSOP2002 (Bretagnon 2002) and DE403 over [1890-2000]. Units are $10^{-10}$ AU for $a, 10^{-10}$ radian for $\lambda$ and $10^{-10}$ for $k, h, q, p$.

\begin{tabular}{lrrrrrr}
\hline \hline Planet & $a$ & $\lambda$ & $k$ & $h$ & $q$ & $p$ \\
\hline Mercury & 0.1 & 2.5 & 0.7 & 0.8 & 0.3 & 0.2 \\
Venus & 0.2 & 6.6 & 1.1 & 0.9 & 0.4 & 2.3 \\
EMB & 0.3 & 5.2 & 2.0 & 3.5 & 1.7 & 4.9 \\
Mars & 6.6 & 152 & 8.8 & 24.4 & 0.8 & 10.8 \\
Jupiter & 31 & 18.2 & 12.9 & 11.5 & 0.8 & 0.5 \\
Saturn & 210 & 38.6 & 20.4 & 21.5 & 1.1 & 1.1 \\
Uranus & 4900 & 259 & 224 & 343 & 29.3 & 8.0 \\
Neptune & 5500 & 389.8 & 291.7 & 341.5 & 2.8 & 26.3 \\
& & & & & & \\
\hline
\end{tabular}

For each planet or asteroid, we introduce the mean mean longitude as a linear function of time $t, \bar{\lambda}_{\mathrm{P}}=\lambda_{\mathrm{P}}^{0}+n_{\mathrm{P}} t$ where $\lambda_{\mathrm{P}}^{0}$ and $n_{\mathrm{P}}$ are the integration constant of the mean longitude and the mean motion of the planet or of the asteroid, respectively. Each elliptic variable, used to describe the motion, is developed in Poisson series of the mean mean longitudes of the perturbed and perturbing objects (the eight major planets and the five asteroids) and of the three Delaunay's angles of the Moon. The series are developed up to degree 8 with respect to time.

Unfortunately Bretagnon did not have time to complete his work. Nevertheless the comparison of his last results to DE403 shows a real progress compared to VSOP2000. Table 1 gives the maximum differences between VSOP2002 and DE403 over [1890, 2000]. VSOP2002 is better than VSOP2000 by a factor of between 3 and 10 for Mercury, Venus and the EMB, and between 1.5 and 3 for Jupiter and Saturn. For Mars, the accuracy is the same because the perturbations by 295 asteroids included in DE403 and DE405 (Standish 1998) are not taken in account either in VSOP2000 or in VSOP2002. However, VSOP2002 is worse than VSOP2000 for Uranus and Neptune because the perturbations from Pluto are not taken into account.

\subsubsection{VSOP2002b, improvement of the VSOP2002 solution}

In the VSOP2002 solution, we have introduced the following improvements:

- We have added the perturbations of Pluto on the outer planets in the form of Poisson series of only one argument, namely a linear function of time, as explained in Simon (2004). With those perturbations, the results of Table 1 are improved by about a factor of 2 for Uranus and 5 for Neptune.

- We have computed the perturbations due to the $\mathrm{J} 2$ of the Sun on the planets Mercury, Venus, the EMB and Mars. They are mainly secular perturbations for the variables $k, h, q, p$. The formulae for computing those perturbations are standard (see, for instance, Lainey 2002; or Pitjeva 2001a). With the value of J2 given by DE405 $\left(2 \times 10^{-7}\right)$, we computed the secular perturbations $\Delta k, \Delta h, \Delta q, \Delta p$ given in Table 2 . These perturbations explain completely the secular
Table 2. Secular perturbations for the variables $k, h, q, p$ of Mercury, Venus, the EMB and Mars due to the J2 of the Sun $\left(2 \times 10^{-7}\right)$. Unit is $10^{-10}$ by 1000 years.

\begin{tabular}{lrrrr}
\hline \hline Planet & $\Delta k$ & $\Delta h$ & $\Delta q$ & $\Delta p$ \\
\hline Mercury & -2459.7 & 547.3 & -192.4 & -306.9 \\
Venus & -6.4 & -5.7 & -41.1 & 11.0 \\
EMB & -6.5 & -1.5 & -24.8 & 6.3 \\
Mars & 3.6 & 8.0 & -4.6 & 0.5 \\
\hline
\end{tabular}

drifts between VSOP2000 and DE405 found by Moisson (2000) for the variables $k, h, q, p$ of Mercury.

- We have introduced the perturbations induced by 295 more asteroids as described in Sect. 2.1.3.

- We have computed new integration constants for the analytical solution by comparison with the JPL numerical integration DE405 (Standish 1998), over [1890, 2000].

The resulting analytical solution, fitted to DE405, is called VSOP2002b.

\subsubsection{Perturbation by the asteroids}

Previous works (Mignot 2002; Williams 1984) have studied asteroid perturbations by analytical methods. In Mignot (2002), the perturbations induced by about 400 asteroids upon Mars orbit were estimated to the first order of mass. For each variable and for each couple Mars-asteroid, the perturbations have the form of a Fourier series of the mean mean longitudes of Mars and of the asteroid.

In the present work, we rather computed the perturbations in a more compact form, based on Poisson series of only one argument, $\mu$, described by Simon (2001). If $n_{\mathrm{j}}$ and $n_{\mathrm{S}}$ denote the mean motions of Jupiter and Saturn, then $\mu$ is given by $\mu=\left(n_{\mathrm{j}}-\right.$ $\left.n_{\mathrm{S}}\right) t / 880=0.359536 t$ where $t$ is given in thousands of years. $\mu$ is a 17485 year period argument. It is also the argument of the Poisson series corresponding to the perturbations by Pluto added to VSOP2002.

We have applied this formulation to the 295 asteroids, besides the 5bigs, chosen by Williams (1984) as the most perturbative asteroids of the Mars orbit. We have computed the perturbations of these asteroids on Mercury, Venus, EMB, Mars and Jupiter as follows. First, we computed by harmonical analysis (Simon 1987) the first order perturbations for each variable and each couple planet-asteroid in the form of Fourier series of the mean mean longitude of the planet and of the asteroid. Each argument of the Fourier series has the form $i \bar{\lambda}_{\mathrm{P}}+j \bar{\lambda}_{\mathrm{A}}$ where $i$ and $j$ are integers and $\bar{\lambda}_{\mathrm{P}}$ and $\bar{\lambda}_{\mathrm{A}}$ are the mean mean longitudes of the planet and of the asteroid, respectively. We write $i n_{\mathrm{P}}+j n_{\mathrm{A}}=q \mu+\epsilon t$ where $q$ is an integer and where $\epsilon$ is as small as possible. Using this relation, we transform the Fourier series of the mean mean longitudes in Poisson series of $\mu$. We add, for each variable of each planet, the Poisson series given by all the asteroids. Finally, we obtain the analytical perturbations of the 295 asteroids in a very simple form: for each variable of the planets Mercury, Venus, the EMB, Mars and Jupiter, these 
Table 3. Secular perturbations for the variables $k, h, q, p$ of Mercury, Venus, the EMB and Mars due to the asteroids given by VSOP2002b and the Numerical integration (NI). The NI drifts are estimated by comparison to DE405, only for Mercury and Venus. Unit is $10^{-10}$ by 1000 years.

\begin{tabular}{l|rr|rr|rr|rr}
\hline \hline Planet & \multicolumn{2}{|c|}{$\Delta k$} & & \multicolumn{2}{|c|}{$\Delta h$} & \multicolumn{2}{|c}{$\Delta q$} & \\
& VSOP & NI & VSOP & NI & VSOP & NI & VSOP & NI \\
\hline Mercury & -42.6 & -47.0 & 8.8 & 9.2 & 7.0 & 10.2 & -11.6 & -14.9 \\
Venus & 2.2 & 0.8 & -12.4 & -14.4 & 10.1 & 17.3 & -6.7 & -8.6 \\
EMB & -6.3 & & -29.4 & & -15.4 & & -4.8 & \\
Mars & 176.4 & & 176.9 & & -5.6 & & -52.8 & \\
\hline
\end{tabular}

perturbations are given by only one Poisson series of only one additional argument $\mu$ (exactly as the perturbations by Pluto) and are then easy to introduce in the VSOP solution. We have computed the series with the precision of VSOP2002, keeping all the terms inducing contributions greater than $0.5 \times 10^{-12} \mathrm{rad}$ over $1000 \mathrm{yr}$. With this accuracy, the series corresponding to the perturbations on the mean longitudes of Venus, EMB, Mars and Jupiter contain 55, 295, 2760 and 1500 terms respectively. For Mercury, there are only secular terms. For Mars, the differences between this new solution, VSOP2002b and DE403 are decreased by a factor of between 2 and 5, depending on the variable, compared to the results shown in Table 1 . The asteroids give also significant secular perturbations $\Delta k, \Delta h, \Delta q, \Delta p$ for all these planets. In the following (see Sect. 2.3), we will see that those secular terms also have been detected by another independant method, the comparison between DE405 and a new numerical integration developed at the IMCCE. Table 3 gives the values of these secular perturbations for Mercury, Venus, EMB and Mars given by the analytical theory and for Mercury and Venus by numerical integration. The asteroids give also secular perturbations of the variable $\epsilon$ related to the mean longitude $\lambda$ by $\lambda=\int n \mathrm{~d} t+\epsilon$ where $n$ is the mean motion. The secular trends produce significant changes in the integration constants of the semi-major axis. For the planets Mercury, Venus, EMB and Mars, these secular perturbations are respectively $-312 \times 10^{-10}$ rad over $1000 \mathrm{yr},-812 \times 10^{-10} \mathrm{rad}$ over $1000 \mathrm{yr}$, $-1420 \times 10^{-10}$ rad over $1000 \mathrm{yr}$ and $-3364 \times 10^{-10} \mathrm{rad}$ over $1000 \mathrm{yr}$. These values are similar to those obtained by Pitjeva (2004). These changes in the semi-major axis have the same effects as changes in the secular part of the mean longitudes and produce significant modifications of the motions of these planets.

\subsection{Numerical integration}

To integrate numerically the planetary equations of motion, we used an algorithm developed by Moshier (1992), based on an Adams-Cowell integrator. Planetary perturbations are computed as described in Newhall et al. (1983) and we added the perturbations induced by the asteroids and by the solar oblateness.

The effects of the Sun J2 were added following the usual formulae (i.e. Sinclair \& Taylor 1985) applied in the plan of the Sun's equator. In Table 2, we present the drifts in the inner planet orbits induced by the introduction of the solar oblateness in the computation of the gravitational potential of the Sun, estimated by analytical analysis. These drifts are the signature
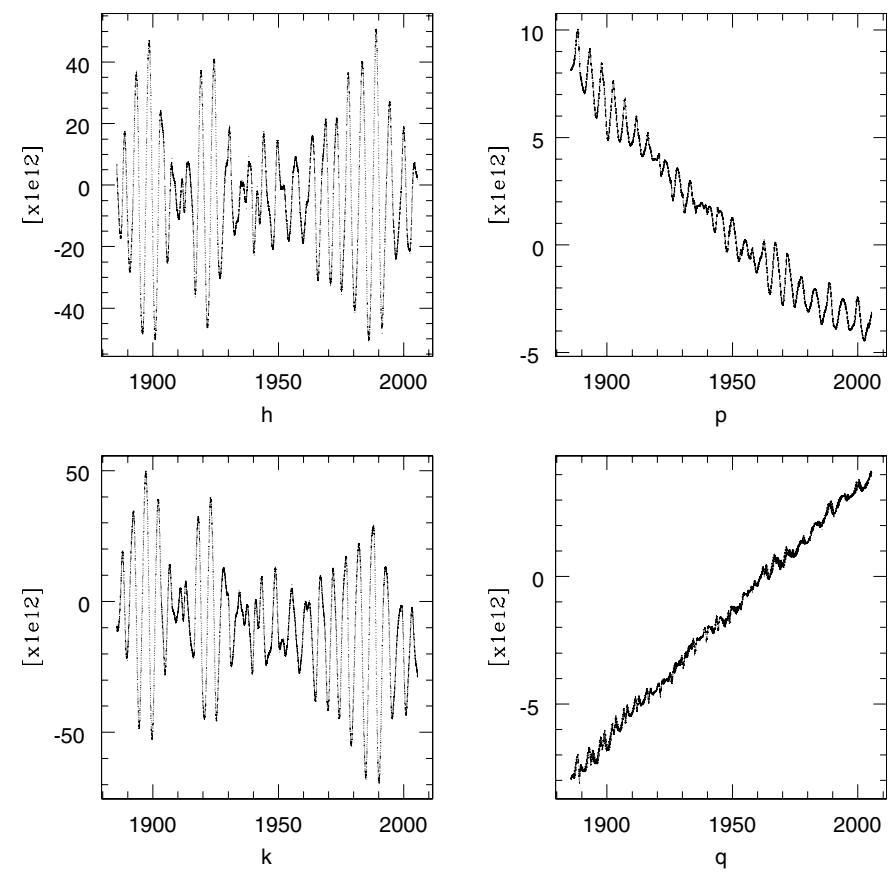

Fig. 1. Differences between our numerical integration without asteroid perturbations included in Mercury orbit and DE405 in $(k, h, q, p)$ for the Mercury orbit. The remaining drifts are quite small compared to those presented in Table 2 and induced by the lack of modeling of the solar oblateness.

of a lack of modeling of the solar oblateness. As one can see in Fig. 1, no important drifts can be detected in the comparison between our numerical solution of the Mercury orbit and DE405 after the introduction of the $\mathrm{J} 2$ perturbations. The remaining drifts are not significant compared to those presented in Table 2.

Concerning the asteroids, the 5bigs are integrated separately from the main integration of the planetary perturbations. The perturbations of the nine planets upon these asteroids are taken into account and integrated using a Burlich-Stoer integrator. From the deduced positions and velocities, Chebychev polynomials are built and used in the main integration, associated with the DE405 asteroid mass determinations, to estimate and include the 5bigs perturbations upon the 9 planets.

Furthermore, we added to the main integration the accelerations upon all nine planets induced by the 295 asteroids selected by Williams (1984). To estimate such accelerations, we used asteroid positions computed from mean orbits (Williams 1984) and asteroid masses deduced from taxonomic densities and diameters given by JPL (Standish 1998). 
The next step of our numerical integration evolution will be its complete fit to space tracking, radar, VLBI and optical observations. The adjustement process is currently being finalysed. However, to simulate such an observational fit, a first preliminary fit was done on the JPL DE405 fitted to observations solution over an interval from 1970 to 2003. This interval represents time during which the most accurate and the most weighted observations are avaliable. It corresponds to the beginning of the Viking tracking data and to the end of the avaliable MGS and Mars Odyssey observations.

\subsection{Comparison to DE405}

We have estimated the impact of the asteroid perturbations by comparison with the JPL DE405 solution. The JPL DE405 solution integrates separately the orbits of Ceres, Pallas and Vesta under their own gravitational forces, those of the Sun, the planets and the Moon. The orbits of the other 297 asteroids (Iris and Bamberga included) are also integrated separately under the gravitational action of the Sun, the planets, Ceres, Pallas, Vesta and the Moon. However, only the force vectors of these 297 asteroids upon Mars and the EMB as well as their contributions to the solar system barycenter were computed and included in the main planetary integration. Thus, there are two kinds of differences between our solutions and DE405. Firstly, we did not compute the orbits of the 300 asteroids in exactly the same way (see Sects. 2.2 and 2.1). Secondly, unlike the DE405 model, we included the asteroid perturbations on all planets and not only on Mars, the EMB and the solar system barycenter.

\subsection{Mars and the EMB}

In DE405, in VSOP2002b and in our numerical integration, the Mars and the EMB orbits have been computed taking into account the perturbations of 300 asteroids. However, the computation of the asteroid orbits used to estimate the perturbations on Mars and the EMB was not done in the same way. In our analytical and numerical solutions, we used mean orbits of 295 asteroids and we integrated numerically and analytically the perturbations of the Sun and the planets over Ceres, Pallas, Vesta, Iris and Bamberga. In DE405, the mutual perturbations of Ceres, Pallas and Vesta were included and the orbits of the 297 other asteroids (Iris and Bamberga included) were integrated numerically, and not only deduced from mean elements as in our solutions.

In Fig. 2, the differences between our numerical integration, VSOP2002b and DE405 are plotted in $k$ and $h$. As one can see, the numerical integration (NI) and VSOP2002b have very similar behaviours compared to DE405. In Fig. 3, the differences in Mars heliocentric distances also show the consistent results of the two solutions relative to DE405. Concerning the EMB heliocentric distances, one can see that the numerical integration gives differences to DE405 2 times smaller than VSOP2002b. Nevertheless, the VSOP2002b shows an accuracy compared to DE405 of about ten meters over 30 years for both the Mars and the EMB orbit. Such precision is quite satisfying for an EMB analytical solution. In Fig. 3, the differences in Mars and EMB
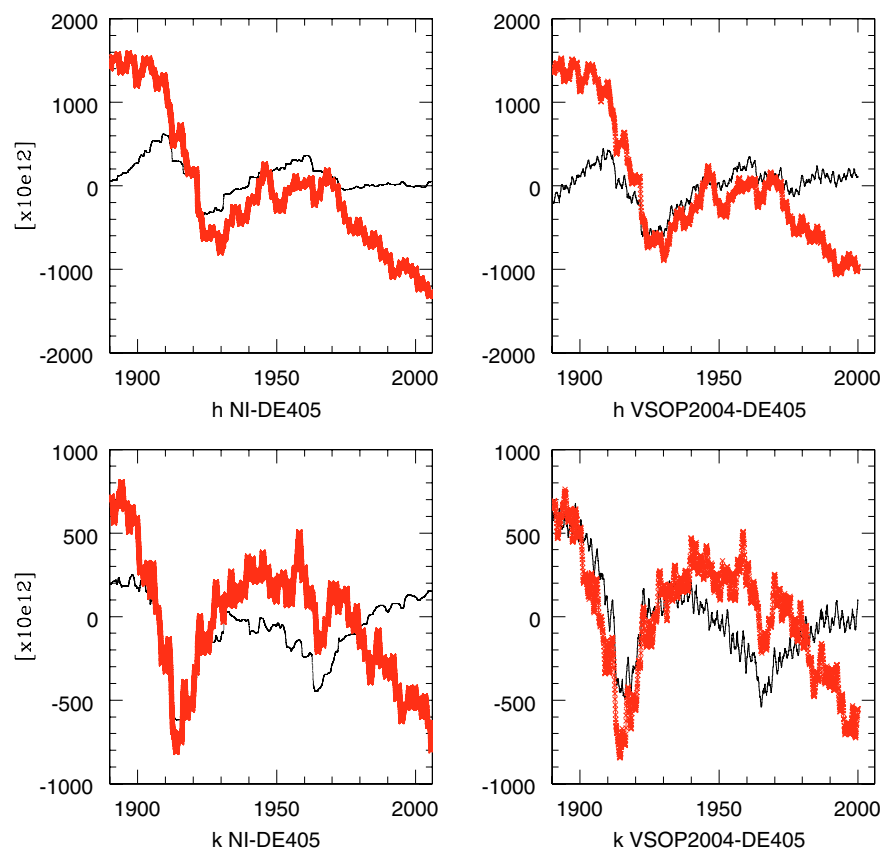

Fig. 2. Differences between our numerical integration (NI) and DE405 (left-hand plots) and VSOP2002b and DE405 (right-hand plots) in $(k, h)$ variables for the Mars orbit. The thick line is for a numerical integration or an analytical solution without the 295 asteroid perturbations. The thin line shows the differences between DE405 and the numerical integration or the analytical solution including the 295 asteroid perturbations.
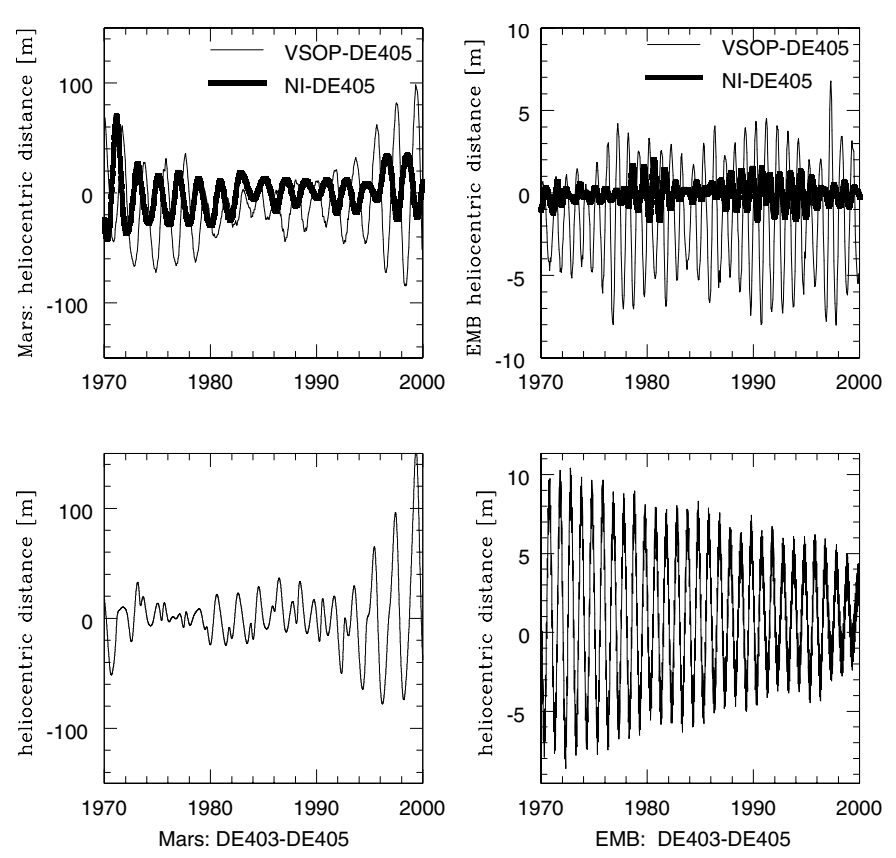

Fig. 3. Differences in meters for the Mars and EMB heliocentric distances between our numerical integration (thick line), VSOP2002b (thin line) and DE405 (upper plots) and DE403 and DE405 (lower plots).

heliocentric distances between our solutions and DE405 are at the same level of magnitude as the previous JPL planetary solutions DE403 compared to DE405. This result is very interesting 

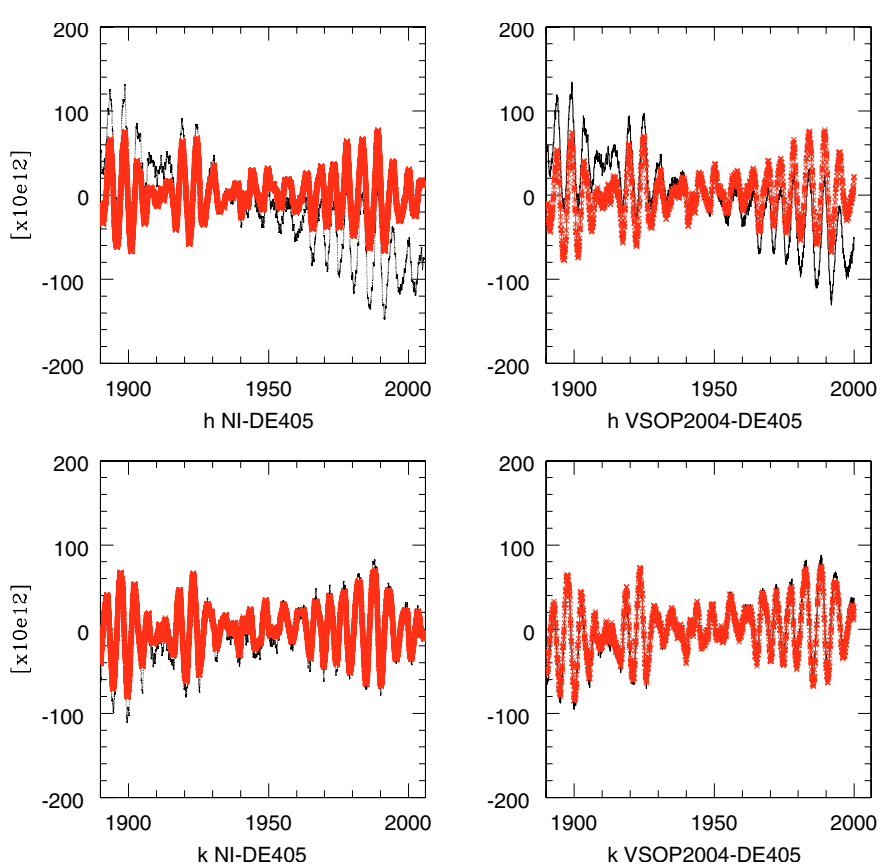

Fig. 4. Differences between our numerical integration (NI) and DE405 (left-hand side plots) and VSOP2002b and DE405 (right-hand side plots) in $(k, h)$ variables for the Venus orbit. The thick line shows differences between DE405 and our numerical integration without the 295 asteroid perturbations included in the Venus orbit. The thin line shows the differences with 295 asteroid perturbations included in our numerical integration of the Venus orbit.

for the analytical solution of the Mars motion. It shows that an analytical modeling of such a complex orbit is possible with very good accuracy. We stress that the accuracy limit of the analytical solution has not yet been achieved. Improvements, especially those induced by the new iteration process will lead to even better accuracy. As it was shown in Pitjeva (2001a) the asteroid orbits computed with the mean elements of 295 asteroids by Williams (1984) over a very long time interval differ significantly from their true orbits, demonstrating the necessity of integrating the orbits of 300 asteroids in the future. Such an improvement has already been implemented in the next version of our numerical solution to be published in 2005 .

\subsection{Mercury and Venus}

The second difference between our solutions and DE405 comes from the introduction of the 295 asteroid accelerations in the computation of the forces upon the Mercury and Venus orbits. Analytically, it was estimated that such an introduction induces drifts in $(k, h, p, q)$ variables of the perturbed orbit. These drifts are gathered in Table 3. The comparisons between our numerical integration and DE405 show such drifts, as one can see in Fig. 4 for the Venus orbit and in Fig. 5 for the Mercury's orbit. In Fig. 4, the numerical integration and the analytical solution again give very close results and it is clear (see Fig. 5) that the numerical integration of the 295 asteroid perturbations upon Mercury orbit introduces important drifts in $(k, h, p, q)$. In Table 2, analytical estimations of the drifts induced by the
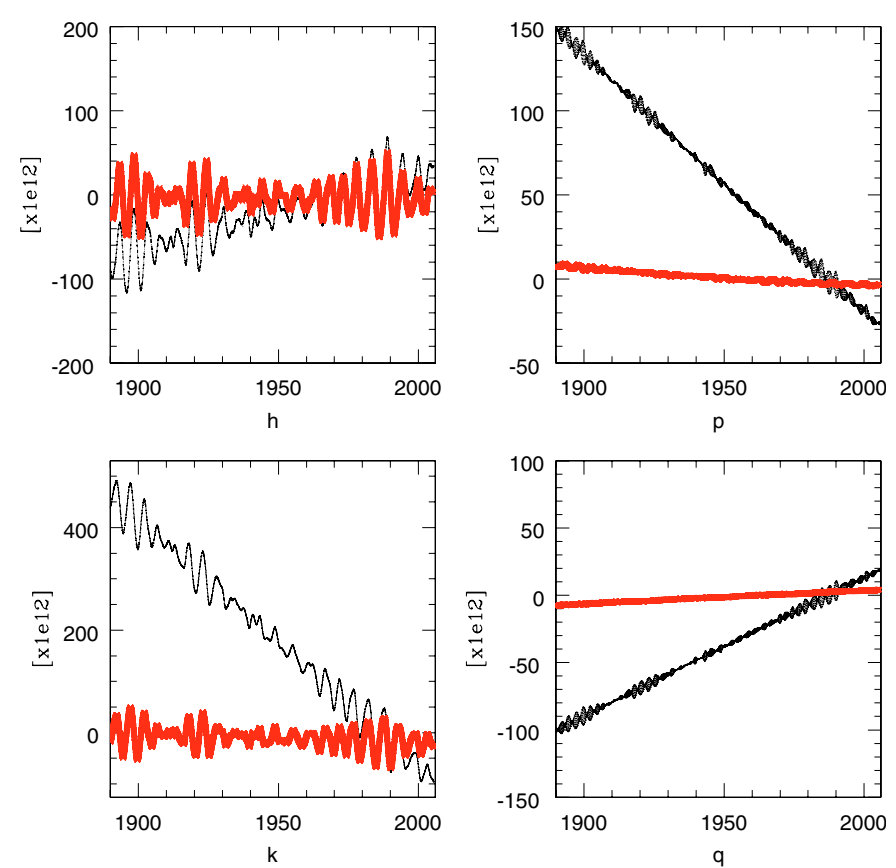

Fig. 5. Differences between our numerical integration and DE405 in $(k, h, q, p)$ for the Mercury's orbit. The thick line shows differences between DE405 and our numerical integration without asteroid perturbations included in Mercury orbit. The thin line shows the differences with the 295 asteroid perturbations included in our numerical integration of the Mercury orbit.

introduction of the 295 asteroid perturbations upon the inner planet analytical orbits can be compared to the drifts obtained by comparison between our numerical integration and DE405. These figures are quite comparable. If we keep in mind that the analytical solution and the numerical integration are two independent methods of orbit computations then these comparisons show that the estimated drifts are real and not due to a mismodeling of the analytical solution or the numerical integration. They also reveal the non-negligible impact of the main belt asteroids on the Mercury and Venus orbits. In Fig. 6, one can see the impact of such perturbations in terms of Mercury heliocentric distances over 30 years.

\section{Discussion}

Based on these results, we can say that with the new models of asteroid perturbations described in Sect. 2.1, VSOP2002b seems to have an accuracy comparable to the best available numerical integrations. It is clear that an analytical solution can produce ephemerides as accurate as about tens of meters on the both Mars and the EMB heliocentric orbits over several decades (Fig. 3). The accuracy limit of such a solution is then improved. In an analytical solution, all perturbations are estimated and introduced individually in the orbit estimation. Therefore, it is a very powerful tool to check numerical integration results as shown here.

An important effect on the Mercury orbit is the influence of the Sun oblateness. Several estimations of the Sun J2 parameter can be found in the literature. Some of these (Pitjeva 2001b) 
Table 4. Maximum Cartesian accelerations over 30 years in $10^{-4}$ meters per day squared upon Mercury, Venus and the EMB induced by the AAA asteroids (left column) and the 295 main belt asteroids.

\begin{tabular}{lll}
\hline \hline Perturbed Planet & $(\ddot{x}, \ddot{y}, \ddot{z})$ induced by & $(\ddot{x}, \ddot{y}, \ddot{z})$ induced by \\
& AAA asteroids & Main belt asteroids \\
& $10^{-4} \mathrm{~m} \mathrm{~d}^{-} 2$ & $10^{-4} \mathrm{~m} \mathrm{~d}^{-} 2$ \\
\hline Mercury & $(-0.006,0.004,-0.003)$ & $(-8,-8,-3)$ \\
Venus & $(0.015,0.002,-0.01)$ & $(-10,10,-4)$ \\
EMB & $(0.015,0.015,-0.015)$ & $(-10,-10,-6)$ \\
\hline
\end{tabular}
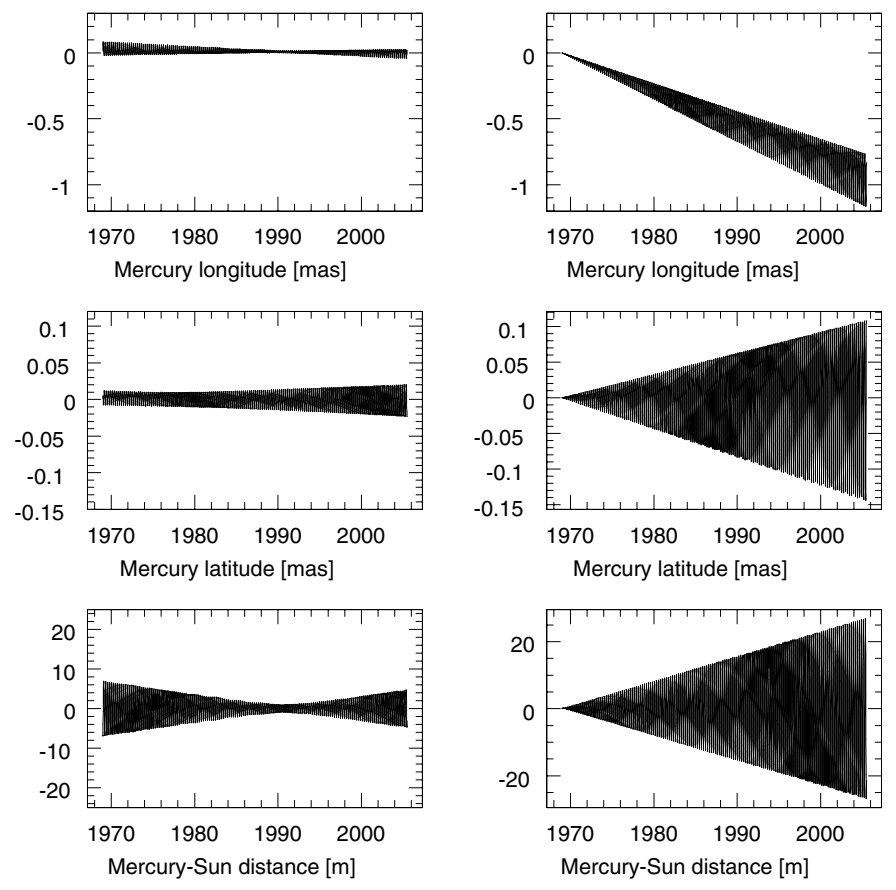

Fig. 6. Differences in heliocentric Mercury longitudes, latitudes and distances between our numerical integration including asteroid perturbations upon Mercury orbit and our numerical integration without (left hand side plots), and between our numerical integration including the perturbations induced by a Sun $\mathrm{J} 2$ equal to $2.0 \times 10^{-7}$ and integration with a $2.1 \times 10^{-7}$ value of $\mathrm{J} 2$ (right hand side plots).

were done considering the effects of the Sun J2 on the Mercury orbit. However, considering the effect of the main belt asteroids on its orbit, one can question if such results are relevant, especially if these estimations are based on planetary solutions that do not consider asteroid perturbations on Mercury's orbit. One may note that the estimations by Pitjeva (2001b) based on the EPM2000 solution were obtained by taking into account the influence of the 300 main belt asteroids upon the orbits of Mercury and all the planets. Furthermore, in a very close future (2007), spacecrafts will be sent to Mercury. We will then have very accurate ranging and VLBI data available for Mercury orbit adjustments and other astronomical constant adjustments such as the Sun oblateness parameters. It will then be interesting to investigate if the perturbations of the main belt asteroids could have an impact on such determinations. We then have tested the possible impact on the Mercury heliocentric longitudes, latitudes and distances if the value of the Sun J2 was changed from $2.0 \times 10^{-7}$ to $2.1 \times 10^{-7}$ in our numerical integration. Figure 6 shows the differences in Mercury heliocentric longitudes, latitudes and distances over 30 years induced by a change of $\mathrm{J} 2$, on the hand, and by the introduction of the 295 asteroid perturbations on the Mercury orbit, on the other hand. As one can see, the impact of the asteroids is not negligible in distances compared to the variations induced by the $\mathrm{J} 2$ change. If a closer analysis is done over the elliptic variables, the 295 asteroid perturbations limit the accuracy of the $\mathrm{J} 2 \mathrm{de}$ termination to about $5 \%$. This shows that the introduction of such perturbations is important if an accurate determination of astronomical constants is to be done based on the analysis of Mercury dynamics.

Finally, we discuss the impact of the Appolo Athen Amor asteroids (AAA) on the Mercury, Venus and the EMB orbits. These asteroids are characterised by their Earth-crossing orbits and more generally by perihelion distances smaller than 1.3 AU and their aphelion distances greater then 0.983 AU (Morbidelli et al. 2002). Until now, the perturbations we have estimated and added to the inner planet models were perturbations induced by a group of 300 main belt asteroids selected as being the most perturbative minor planets for the Mars orbit. No one has considered different asteroids from a Mercury, Venus and the EMB point of view. Even if a very few diameter or bulk density estimations exist for the AAA asteroids, we have computed the accelerations induced by a set of 2649 AAA asteroids extracted from the Bowell asteroid database (2003). We have supposed a diameter of $0.5 \mathrm{~km}$ for AAA asteroids with unestimated diameters. This diameter hypothesis is a largely overestimated value. To compute the asteroid masses, we used an hypothesis of $1.8 \mathrm{gm} / \mathrm{cm}^{3}$ for the bulk density, which corresponds to the lowest bulk density value estimated by JPL DE405. In Table 4, we have gathered the accelerations of Mercury, Venus and the EMB orbits induced by the AAA asteroids (second column) and the accelerations induced by the 295 main belt asteroids (third column) we considered previously. It appears that the AAA accelerations are several order of magnitudes smaller than the accelerations induced by the main belt asteroids. In consequence, these asteroids seem to be quite negligible for the inner planet short term dynamics. 


\section{Conclusion}

Two results can be stressed from this paper. Firstly, we have presented the first results obtained with the new VSOP analytical solution, VSOP2002b. In this solution, perturbations induced by Pluto, the Sun oblateness and 300 asteroids were introduced. This solution VSOP2002b is almost as accurate as the most precise numerical planetary ephemerides available, DE405. We will be able to improve VSOP2002b by taking into account the change of the integration constants upon all the terms of the analytical development. Such an improvement is done by recomputing the entire solution with the new constants. The new solution, based on this final iteration, will be called VSOP2004.

Secondly, we have shown that the perturbations induced by the main belt asteroids upon Mercury and Venus orbits are non negligible, especially in the perspective of future Mercury orbiter space missions. We obtain this result by developing our own numerical solutions of motion and by comparison between VSOP2004, our numerical solution and the JPL DE405 solution.

Based on this result, it would be interesting in follow-up studies to investigate the relevance of the choice list of the most perturbative asteroids of Mars done by Williams (1984). What happens to such a list if we want to consider also the most perturbative asteroids on the Mercury, Venus and the EMB orbits? What is the impact of such a modification on the determination of astronomical constants such as the astronomical unit or the Sun J2? Such questions will be of importance for future Mercury space missions.

Acknowledgements. We wish to thank M. Gastineau for his help with the installation and the modifications provided to the old version of the Mosheir C-program. We also wish to thank Dr. E. Pitjeva for her useful comments and suggestions.

\section{References}

Bretagnon, P. 2002, private communication

Krasinsky, G. A., Pitjeva, E. V., Vasiley, M. V., \& Yagudina, E. I. 2002, Icarus, 158,98

Lainey, V. 2002, Théorie dynamique des satellites galiléens, Thèse de doctorat de l'observatoire de Paris

Mignot, S. 2002, Exploration des perturbations de la ceinture principale d'astéroïdes sur Mars, Stage de DEA de l'observatoire de Paris

Moisson, X. 2000, Intégration du mouvement des plantes dans le cadre de la relativité générale, Thèse de doctorat de l'observatoire de Paris

Moisson, X., \& Bretagnon, P. 2001, Celest. Mech. Dyn. Astr., 80, 205

Morbidelli, A., Bottke, Jr., W. F., Froeschlé, Ch., \& Michel, P., in ASTEROIDS III, ed. Bottke Jr., W. F., Cellino, A., Paolicchi, P., \& Binzel, R. P., 409

Moshier, S. L. 1992, A\&A, 262, 613

Newhall, X. X., Standish, E. M., \& Williams, J. G. 1983, A\&A, 125, 150

Pitjeva, E. V. 2004, Private communication

Pitjeva, E. V. 2001a, Celest. Mech. Dyn. Astr., 80, 249

Pitjeva, E. V. 2001b, A\&A, 371, 760

Sinclair, A. T., \& Taylor, D. B. 1985, A\&A, 147, 241

Simon, J. L. 1987, A\&A, 175, 303

Simon, J. L. 2001, Notes scientifiques et techniques de l'IMCCE S076, 77

Simon, J. L. 2004, Notes scientifiques et techniques de l'IMCCE S081, to be published

Standish, E. M. 1998, JPL planetary and lunar ephemerides, DE405/LE405, JPLIOM 312.F-98-048

Standish, E. M., Newhall, X. X., Williams, J. G., \& Folkner, W. F. 1995, JPL planetary and lunar ephemerides, DE403/LE403, JPLIOM, 314, 10

Standish, E. M., \& Fienga, A. 2002, A\&A, 384, 322

Williams, J. G. 1984, Icarus, 57, 1 